\title{
Influence of the Cholinergic System on the Immune Response of Teleost Fishes: Potential Model in Biomedical Research
}

\author{
G. A. Toledo-Ibarra, ${ }^{1}$ A. E. Rojas-Mayorquín, ${ }^{2,3}$ and M. I. Girón-Pérez ${ }^{1}$ \\ ${ }^{1}$ Universidad Autónoma de Nayarit (UAN), Secretaría de Investigación y Posgrado, Laboratorio de Inmunotoxicología, \\ Boulevard Tepic-Xalisco s/ $n$, Cd de la Cultura Amado Nervo, 63190 Tepic, Nayarit, Mexico \\ ${ }^{2}$ Departamento de Ciencias Ambientales, Instituto de Neurociencias, Centro Universitario de Ciencias Biológicas y Agropecuarias \\ (CUCBA), Universidad de Guadalajara (UdeG), Francisco de Quevedo 180, Col. Arcos Vallarta, 45100 Guadalajara, Jal, Mexico \\ ${ }^{3}$ Departamento de Investigación Básica, Instituto Nacional de Geriatría (INGER), Periférico Sur No. 2767, \\ Col. San Jerónimo Lídice, Del. Magdalena Contreras, 10200 México, DF, Mexico
}

Correspondence should be addressed to M. I. Girón-Pérez; ivan_giron@hotmail.com

Received 26 July 2013; Revised 24 September 2013; Accepted 26 September 2013

Academic Editor: Marco Antonio Velasco-Velázquez

Copyright ( 2013 G. A. Toledo-Ibarra et al. This is an open access article distributed under the Creative Commons Attribution License, which permits unrestricted use, distribution, and reproduction in any medium, provided the original work is properly cited.

\begin{abstract}
Fishes are the phylogenetically oldest vertebrate group, which includes more than one-half of the vertebrates on the planet; additionally, many species have ecological and economic importance. Fish are the first evolved group of organisms with adaptive immune mechanisms; consequently, they are an important link in the evolution of the immune system, thus a potential model for understanding the mechanisms of immunoregulation. Currently, the influence of the neurotransmitter acetylcholine (ACh) on the cells of the immune system is widely studied in mammalian models, which have provided evidence on ACh production by immune cells (the noncholinergic neuronal system); however, these neuroimmunomodulation mechanisms in fish and lower vertebrates are poorly studied. Therefore, the objective of this review paper was to analyze the influence of the cholinergic system on the immune response of teleost fish, which could provide information concerning the possibility of bidirectional communication between the nervous and immune systems in these organisms and provide data for a better understanding of basic issues in neuroimmunology in lower vertebrates, such as bony fishes. Thus, the use of fish as a model in biomedical research may contribute to a better understanding of human diseases and diseases in other animals.
\end{abstract}

\section{Immune System in Teleost Fishes}

Fishes are the phylogenetically oldest vertebrate group and appeared $>560$ million years ago. This group includes $>27,000$ species, representing more than one half of the vertebrates on the planet. The vast majority of fishes are teleosts (teleostei, possessing a bony skeleton) and some are noted for their ecological and economic importance, whereas other species are widely used as biological models for genomic studies and developmental biology $[1,2]$. In addition, because these organisms are the first that present adaptive immune mechanisms (Figure 1), the Big Bang of Immunology [3], the study of the immune system of these organisms is of great relevance because it provides information on the evolution of the immune system in vertebrates, thus supporting the understanding of basic aspects of immunology, therefore the possible treatment of emerging diseases in humans and in other animals [4].

1.1. Lymphoid Organs. Fishes, unlike mammals, lack lymph nodes and bone marrow [5]. However, the anterior kidney or pronephros, analog evolutionary of the bone marrow, possesses important hematopoietic functions (precursor hematopoietic cells appear after $96 \mathrm{~h}$ as postfertilized (hpf) in mesonephric tubules) and also presents similar functions to those of the adrenal gland of mammals, which is key in connections among the neuroimmune-endocrine systems [6-8]. Additionally, the spleen is the main secondary lymphoid organ in fish and presents a significant number of $\left(\operatorname{IgM}^{+}\right.$B) lymphocytes, in addition to participating in the induction of adaptive immune responses, and is important for the elimination of immune complexes [4]. Regarding 


\begin{tabular}{ccc}
\hline & Innate & Adaptative \\
\hline Humoral & Antimicrobial peptides & Antibodies: \\
& Lectins & IgM \\
& Lysozyme & IgD \\
& Complement & IgT/IgZ \\
\hline Cellular & Macrophages & B-lymphocytes \\
& Neutrophils & T-lymphocytes \\
& Eosinophils & \\
\hline
\end{tabular}

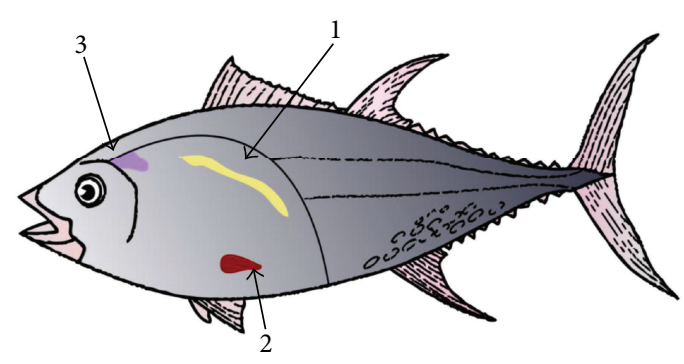

FIGURE 1: Main humoral, cellular, and anatomical components of the immune system in fishes. Fish lymphoid organs: pronephros (1), spleen (2), and thymus (3).

the thymus, this is a bilobed organ localized in the opercular cavity; it is the major site for T-cell development in fish, as well as in mammals, and presents an involution, except that this phenomenon is greatly influenced by hormonal cycles and environmental changes in the latter [9].

1.2. Innate Immunity. With respect to the mechanisms of innate immunity, these are important in early defense against pathogen infection [10] and also play an instructor role in the induction of the adaptive response [4]. The innate humoral components that are mainly characterized in fishes are antibacterial peptides, lysozymes, lectins, acute-phase proteins, and molecules of the complement system (Figure 1) [11-15].

The cells of the innate immune system are activated by Pathogen recognition receptors (PRR), an important type of these are the Toll-like receptors (TLR). In fishes, it has been described that the majority of TLR are present in mammals (TLR1, TLR2, TLR 3, TLR4, TLR5, TLR7, TLR8, and TLR9). In addition, in fishes the presence of some TLR that has not yet been identified in mammals (TLR18-TLR23) has been described. Additionally, in channel catfish (Ictalurus punctatus), the presence has been identified of TLR25 and TLR26, which are apparently present only in fishes $[16,17]$. Finally, there are other TLRs, at least eight more, which have been described in other taxa but not in fishes.

The cells of the innate immune response mainly characterized in fishes are the macrophages, neutrophils, and eosinophils [16]. Macrophages are phagocytic cells that are important in early antimicrobial responses; it is been suggested that the phagocytic process in fish is more effective than in murine models when both are compared [18]. Additionally, neutrophils are the first cells to migrate to the site of infection; these cells possess a highly bactericidal capacity through the release of proteolytic enzymes, antimicrobial peptides, and reactive oxygen species (ROS). In addition, the ability that has been recently demonstrated, in fish neutrophils is that of releasing neutrophil extracellular traps (NET), which are complex structures consisting of DNA, histones, and proteins from granules. These structures are responsible for trapping and extracellular killing of bacteria, fungi, parasites, and also for inactivating viruses [19]. Thus, it has been demonstrated that NETs production can be ROSdependent or -independent. Finally, fish eosinophils, as in mammals, release cytoplasmic granules against extracellular parasites [20, 21].

1.3. Adaptive Immunity. Adaptive immunity mechanisms in fishes play a vital role in protection against recurrent infections through the generation of cellular and humoral mechanisms, which generate immunological memory, mediated by T- and B-lymphocytes and antibodies [22]. Fishes are the first vertebrates in which clonal selection and genetic rearrangement in lymphocyte receptors presents [4].

In fishes, leukocytes have been reported with T-cell activity, similar to that of the T-helper and cytotoxic cells of mammals; also, in some species of fish, some structurally conserved cellular markers are present, including Clusters of differentiation (CD) $3^{+}$and T-cell receptors (TCR). Furthermore, based on the profile of cytokines, these also possess T-cell subpopulations similar to those reported in mammals [23].

$\mathrm{B}$-cells are characterized by the expression of antigen receptors (B-cell antigen receptors (BCR)) in the membrane. In teleost fish, B-cells activated, plasmablasts, and plasma cells were identified, which were differentiated from each other mainly by their ability in the production of antibodies $[24,25]$.

Soluble antibodies that have been identified in fishes are primarily (IgM), which are tetrameric and present in high concentrations in plasma and IgD, which, as in mammals, are expressed on the surface of B-lymphocytes. Other antibody isotypes have been identified in fishes, including $\operatorname{IgT}$ and IgZ, which are present mainly in the mucosa, such as in intestine, skin, and gills $[26,27]$. Innate and adaptive mechanisms in fishes, similar to those occurring in mammals, are regulated and interconnected by cytokines. Of these, interleukin $1 \beta$ (IL- $\beta$ ), IL- 6 and tumor necrosis factor alpha (TNF- $\alpha$ ) have been well characterized in these organisms. Other cytokines reported in fish include IL-2, IL-4, IL-8, IL-10, IL-12, and tumor growth factor beta (TGF- $\beta$ ) [2830]. In addition to cytokines, the immune system of teleost fishes is regulated by neuroendocrine interactions, primarily through the hypothalamic-pituitary-interrenal (HPI) axis, because the pronephros, in addition to their roles as lymphoid organs, has important endocrine functions. However, there is also evidence of the effect of hypothalamic-pituitary-thyroid 


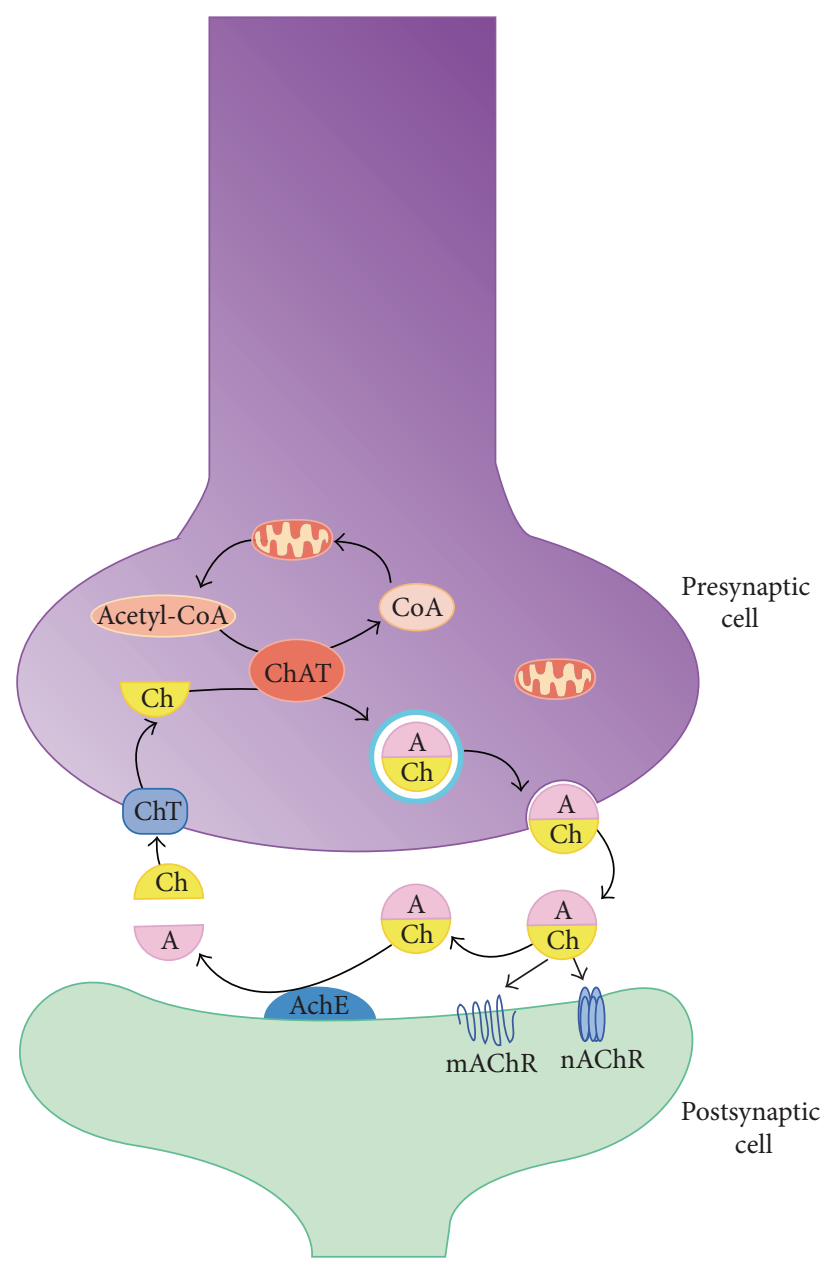

FIGURE 2: Synthesis of acetylcholine in synapse. A: Acetyl; AChE: acetylcholinesterase; Ch: choline; ChAT: acetylcholine transferase; ChT: choline transporter; CoA: coenzyme A; mAChR: muscarinic receptor; nAChR: nicotinic receptor.

(HPT) and the brain intercommunication-pituitary-gonadal axes on the immune response in teleost fish [31].

\section{Cholinergic System in Teleost Fish}

Acetylcholine (ACh) is a neurotransmitter that widely distributed in the central and peripheral nervous systems. It is synthesized from choline and acetyl-Coenzyme A (acetylCoA) by the enzyme choline acetyltransferase (ChAT) and then is stored in presynaptic vesicles until the cell is activated (Figure 2). When ACh is released into the nerve synapse, this neurotransmitter binds two distinct receptors on the postsynaptic cell: the ionotropic nicotinic acetylcholine receptor (nAChR) and the metabotropic muscarinic acetylcholine receptor (mAChR), which are bonded to $\mathrm{G}$ protein. In the synaptic cleft, $\mathrm{ACh}$ is hydrolyzed by the enzyme acetylcholinesterase (AChE) into choline and acetate; approximately $50 \%$ of the choline hydrolyzed is recovered by the high-affinity presynaptic transporter, which achieves continuous production and releases neurotransmitters [35].
There are evidences that ACh is expressed in bacteria, algae, protozoa, and primitive plants, suggesting an early onset of ACh in evolution. These cells utilize ACh as a neurotransmitter. In fish, the following are some functions that have been linked with this neurotransmitter: visual response of optical circuits; gustatory information processing during feeding, and the processing of motor information $[36,37]$.

To understand the teleost cholinergic system, it is relevant to identify some important anatomical data about their sympathetic nervous system (SNS), which appears to have particularly unique evolutionary traits for engaging in sophisticated underwater life. This system in teleosts consists of sympathetic ganglia associated with the corresponding spinal nerves, a pair of sympathetic trunks connecting the sympathetic ganglia and the splanchnic nerves. A unique feature of the teleost SNS is that the sympathetic trunks extend into the cranial region and connect several cranial sympathetic ganglia that are associated with the cranial (trigeminal, facial, glossopharyngeal, and vagal) nerves. Organization of the cranial sympathetic ganglia varies among species [38]. In many teleost species, a pair of celiac ganglia is present at the point where the celiac arteries emerge from the aorta. The postganglionic fibers emerging from the celiac ganglia are distributed to the coelomic organs, along with the celiac artery. The celiac ganglia are connected to the sympathetic trunk via the splanchnic nerves.

In mammals, the sympathetic preganglionic neurons (SPN) are clustered in discrete nuclear columns. Developmental studies have demonstrated that SPN, together with the somatic motor neurons, differentiate from a common primitive motor column [39]. In teleosts, the majority of SPN in the sympathetic ganglia appears to be adrenergic; however, a population of ganglion cells $(<1 \%)$ in the cranial sympathetic ganglia are positive for ChAT; thus, these might be cholinergic [40]. On the other hand, AChE-positive neurons are observed in the periaqueductal gray (PAG) (central gray) in some species of teleost fish, and ChAT-positive neurons were not found dorsally to the central canal but scattered in the lateral region of the central gray [38].

In a comparative study of four fish types, it was observed that distribution of the forebrain cholinergic cells is markedly different among species, suggesting that some structures appeared after the cholinergic system, while in the brainstem, cholinergic structures are well preserved during evolution [41]. The developmental pattern of ChAT-positive neurons has been described in the zebrafish; Arenzana et al. (2005) mentioned that during this fish's development, these neurons are detectable in the forebrain and in the mesencephalic tegmentum region, from $60 \mathrm{hpf}$, while in the optic tectum, the midbrain does not appear until hatching. In the cerebellum, these cells were observed in the isthmic region and medulla oblongata at the end of the embryonic life. Finally, in the spinal cord, motoneurons are detected from $48 \mathrm{hpf}$ [32].

Several studies have shown that the organization of the cholinergic system in the central nervous system (CNS) is similar among vertebrates; in fishes, however, there is greater variability [42]. Mueller et al. (2004) note the presence of ChAT-positive neuronal cells in different regions of the zebrafish brain, for example, in the telencephalon, preoptic 


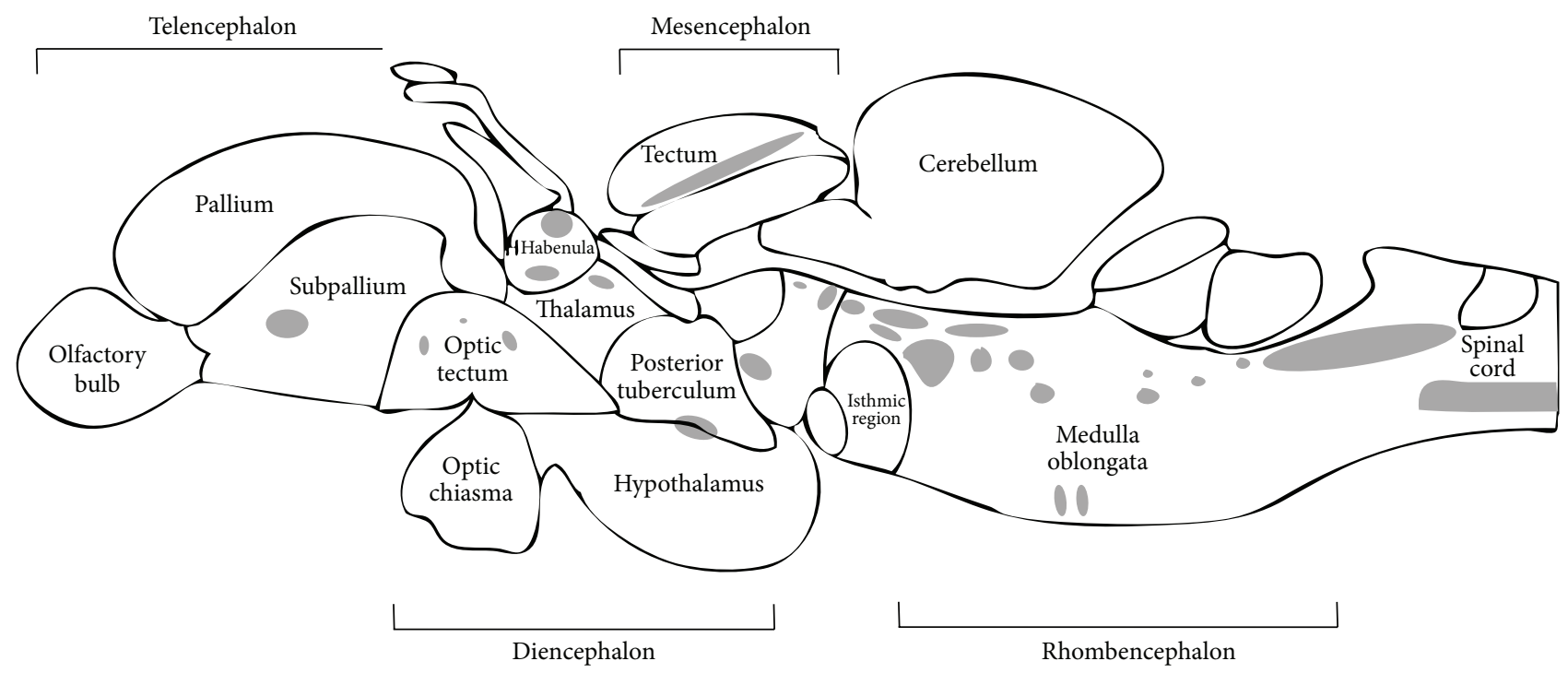

Figure 3: Schematic illustration of location of central nervous cholinergic neuronal populations (gray regions) in adult zebrafish brain (adapted from [32]).

region, diencephalon, mesencephalon, isthmic region, and rhombencephalon [43] (Figure 3).

The nicotinic acetylcholine receptor $(\mathrm{AChR})$ is an integral protein of the postsynaptic membrane that has been studied since the 1970s, first in fishes (as in Electrophorus spp. and Torpedo spp.) and later in mammals (from mammalian muscle). $\mathrm{mAChR}$ are related to neurotransmission, neuromodulation, and olfactory mechanisms, while $\mathrm{nAChR}$ are involved in glutamate release and memory construction; both receptors have been characterized in zebrafish [44]. Steele et al. (2007) [45] suggest a role for the $\mathrm{mAChR}$ in regulating the heart rate under hypoxia in zebrafish larvae, while the function of the $\mathrm{nAChR}$ receptor was elucidated by exposing zebrafish to low doses of nicotine, causing effects on the memory of the fish, in addition to anxiolytic effects, as evidenced by swimming upright $[46,47]$.

Regarding the characterization of the AChE enzyme, this has been identified in brain tissue of various tropical fish, such as pirarucu (Arapaima gigas), cobia (Rachycentron canadum), and Nile tilapia (Oreochromis niloticus) [48]. Employing immunohistochemical techniques, Clemente et al. (2004) observed AChE-positive neurons in the olfactory bulb and the telencephalon and the diencephalon region remains the least dense in AChE-positive neurons; these were more abundant in the isthmic region and in medulla oblongata subdivisions [37].

\section{Cholinergic Influence on the Immune System in Teleost Fish}

Currently, the influence of the nervous system on the immune system cells is clear. Thus, there is abundant evidence of the effect of catecholamine, cortisol, and opioids, even serotonin, on the immune response in teleost fish. However, research focused on the study of the effect of the cholinergic system on the immune response of these organisms is very limited [49]. Classical cholinesterase enzymes such as AChE and butyrylcholinesterase (BChE) are sensitive to other neurotransmitters such as serotonin; thus, it could well represent an interface for a crosstalk between these neurotransmitter systems $[50,51]$.

The two types of cholinesterases (ChE) are present in vertebrates; AChE and BChE exhibit an aryl acylamidase activity (AAA), which is effectively inhibited by cholinergic and serotonergic agents (ACh, specific anticholinesterase drugs and serotonin) $[50,51]$. Because the serotoninergic system is involved in pathologies such as anxiety and depression, which in turn influences their immunological responses and cholinergic and that serotonergic drugs sensitively inhibit AAA activity, this could represent a point of crosstalk between the cholinergic and serotoninergic systems. However, due to the complexity of these systems and the lack of precise knowledge that continues to prevail with respect to the brain activity of BChE and also concerning the BChE gene itself that appears to have been lost in some fish lineages [52]. This is why the study of the effect of cholinergic components on neuroimmunomodulation is complex; therefore, addressing a more profound analysis of the relationship between these two systems merits a separate discussion [53].

Although the mechanisms of neuromodulation for the cholinergic system in mammals have been extensively elucidated, investigations of this intercommunication in fish are scarce. In this regard, one of the first reports was conducted by Flory (1990) and Flory and Bayne (1991), who demonstrated, in rainbow trout (O. mykiss), that carbachol (a cholinergic agonist) significantly increases the number of antibodyproducing cells and the concentration of ROS in leukocytes $[54,55]$.

Related studies on this fish's spleen structure have shown that this lymphoid organ presents an autonomic innervation. 


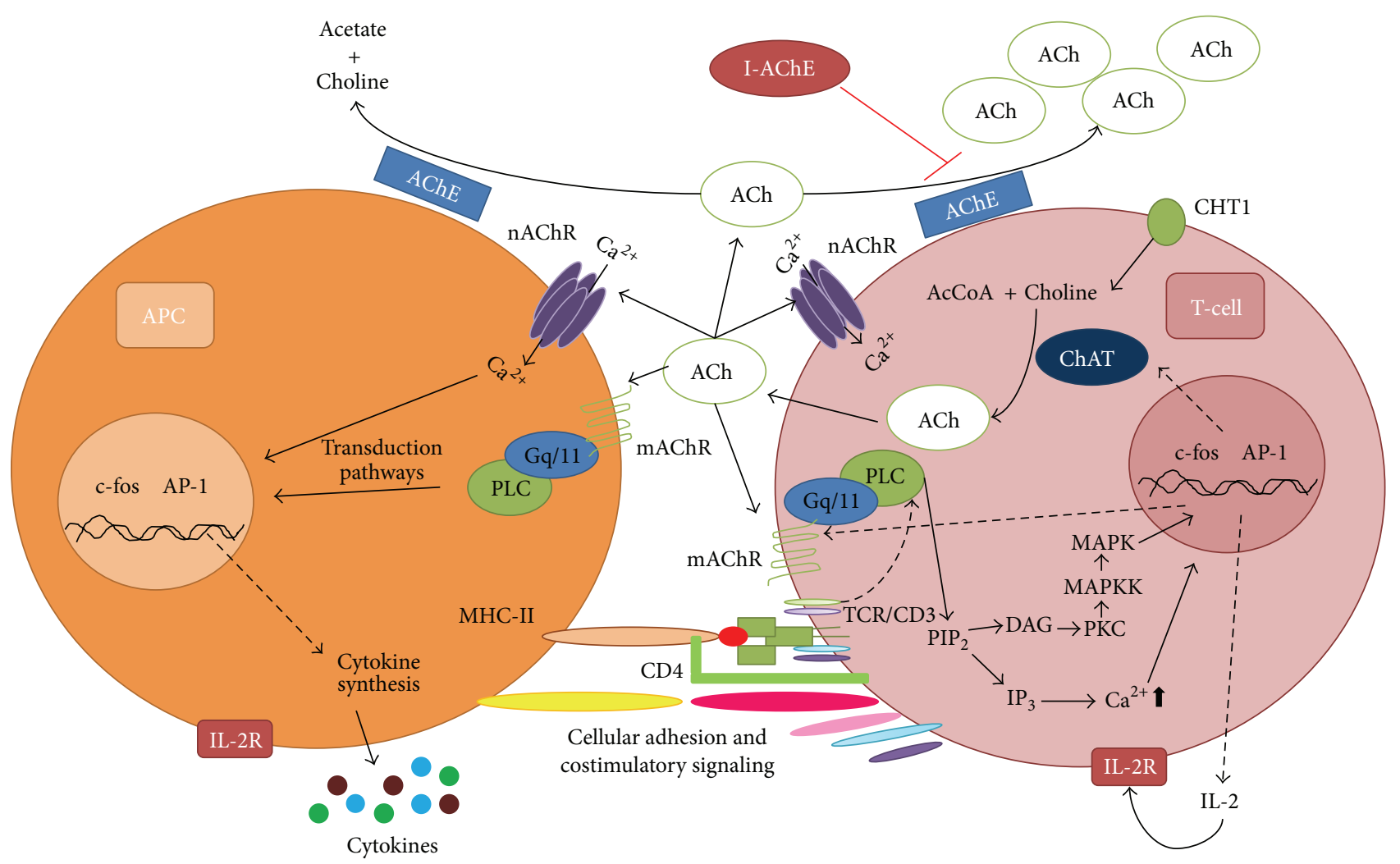

FIGURE 4: Cholinergic system in cells of the immune system and the effect of acetylcholinesterase inhibitors in mammals. AcCoA: acetyl coenzyme; Ach: acetylcholine; AChE: acetylcholinesterase; AP1: activator protein 1; APC: antigen presenting cell; ChAT: choline acetyltransferase; CHT1: high-affinity choline transporter; DAG: diacyl glycerol; I-AChE: acetylcholinesterase inhibitor; IL-2: interleukin 2; $\mathrm{IP}_{3}$ : inositol-1,4,5-trisphosphate; mAChR: muscarinic ACh receptor; MAPK: mitogen activated protein kinase; MAPKK: MAP kinase kinase; MHC II: major histocompatibility complex class II; nAChR: nicotinic ACh receptor; PIP $_{2}$ : phosphatidylinositol 4,5-bisphosphate; PKC: protein kinase C; PLC: phospholipase C; TCR: T-cell receptor (adapted from [33, 34]).

Work on the atlantic cod (Gadus morhua) has revealed that the teleost spleen receives cholinergic nervous input through a branch of the anterior splenic nerve [56]. Experiments in tench fish or dog fish (Tinca tinca) and atlantic cod have shown that exposure to $\mathrm{ACh}$ induces a significant reduction of splenic tissue, while exposure to atropine reversed this effect [57].

Moreover, numerous studies have shown that anticholinergic substances (AChE activity inhibitors), such as organophosphorus pesticides (POF), are able to modulate the immune response of fish, leading in the majority of cases to its immunosuppression $[33,34,58]$. Notwithstanding this, the mechanism of immunotoxicity of these compounds remains unclear. Studies by our research group have shown that exposure, in vivo, of Nile tilapia (O. niloticus) to diazinon (an AChE inhibitor) reduces the proliferative capacity of splenocytes and increases ACh concentration in the spleen, while in vitro exposure to this pesticide or to diazoxon (main metabolite of diazinon) does not affect lymphoproliferation. However, lymphocytes exposed to ACh exhibited reduced lymphocyte proliferation $[59,60]$, suggesting a possible effect of the POF immunotoxicant through alterations in neuroimmunomodulation through cholinergic pathways (Figure 4).
3.1. Nonneuronal Cholinergic System and Lymphocytes. ACh is a major neurotransmitter and its presence has been demonstrated not only in neuronal tissue, but also in prokaryotic and eukaryotic cells, from bacteria to mammalian cells, suggesting the presence of this molecule along evolution [36].

In mammals, the presence has been demonstrated of ACh in extraneuronal tissue, including gastrointestinal epithelium, respiratory, urogenital, placental, and vascular endothelial cells and lymphocytes [61]. ChAT enzyme is constitutively expressed in virtually all cells. In nonneuronal cells, ACh is synthesized and released continuously, in small quantities, into the extracellular environment to maintain cellular homeostasis and to regulate basic cellular functions such as mitosis, differentiation, cytoskeleton organization, and cellular interactions [35].

In terms of immune system cells, it has been demonstrated that these possess, in their membrane, muscarinic $(\mathrm{mAChR})$ and nicotinic (nAChR) receptors, through which it regulates their function [61-64]. Furthermore, ChAT enzyme expression in $\mathrm{CD}_{4}^{+}$and $\mathrm{CD} 8^{+}$T-cells has been confirmed, suggesting that lymphocytes possess all of the necessary biochemical machinery to produce this neurotransmitter, thereby regulating their functions in an autocrine manner 
[65]. Furthermore, in vitro studies with human mononuclear cells have reported the presence of concentrations of ACh in $0.3 \mathrm{pmol} / 10^{6}$ cells, with a synthesis capacity of $2.90 \pm$ $0.84 \mathrm{nmol} / \mathrm{mg}$ protein/h [66].

Experimental data obtained by means of in vitro models and in the absence of neuronal innervation have shown ChAT production in $\mathrm{B}$ cells, macrophages, and dendritic cells from mouse; production of this enzyme appears to be upregulated by TLR activation, a pathway through MyD88 [65]. Moreover, Neumann et al. in 2007 [66] showed, in human leukocytes, that antagonists of nicotinic and muscarinic receptors (tubocumarin and atropine, resp.) significantly decreased the phagocytic functions of granulocytes, but did not change the migration of these cells, whereas in Jurkat cells (the human helper T-lymphocyte leukemic line), exposure to oxotremorine-M (Oxo-M), a cholinergic agonist, significantly increases the synthesis of IL-2, which could be related with transcriptional factor activator protein1 (AP-1) and mitogen-activated protein kinases (MAPK) [67], while in experiments in MOLT-3 cells (the human T-cell leukemia line), the involvement has been suggested of the protein kinase $\mathrm{C}(\mathrm{PKC})$ signaling pathway-MAPK, cyclic adenosine $3^{\prime}, 5^{\prime}$-monophosphate (cAMP), and calcineurin in the synthesis of ACh [64] (Figure 4).

In general, according to the data obtained in mammalian models, it has been proposed that cholinergic activity increases as a result of direct contact between TCR/CD3 molecules, CD4 and CD8 coreceptors, and other accessory molecules [63]. However, cholinergic component data and extraneuronal cholinergic neuroimmunomodulation mechanisms in fish are scarce.

\section{The Cholinergic System in Fish: Another Approach in Biomedicine}

The cholinergic system, in addition to its exerting a significant influence on the functioning of the immune system of vertebrates, is also essential for homeostasis of the organism. Cholinergic components are related to physiological changes caused by insecticides, poisons, and chemical weapons, as well as by human degenerative diseases such as myasthenia gravis and Alzheimer's disease (AD) [68].

Therefore, besides the study of cholinergic influence on the immune system of fishes, there is now a growing interest in developing new biological models that permit the study of neuromodulation. Among the species of fish that have been employed in this aspect, we find Pacific electric ray (Torpedo californica), eel (Electrophorus electricus), goldfish (Carassius auratus), Nile tilapia (O. niloticus), and zebrafish (Danio rerio) [69-72].

Myasthenia gravis is an autoimmune disease in which autoantibodies are generated against a cholinergic receptor. Research utilizing fishes as a model have been prominent in the study of this disease. Some species, such as T. californica, have electric organs whose function is dependent on the cholinergic system; thus, these organisms are a rich source of AChR, molecules that have been investigated to determine the epitopes related to the development of this disease [73].
Another disease in which the use of fish is proposed as a study model is $\mathrm{AD}$, most common form of dementia and which is characterized mainly by massive neuronal loss and impaired synaptic processes localized in the cerebral cortex, particularly in the frontal and temporal lobes and the hippocampus. AD is related to cholinergic system dysfunctions, such as the loss of cholinergic neurons in the basal forebrain and the hippocampus. In this regard, the effect of various cholinergic drugs has been evaluated in zebrafish and it has been reported that scopolamine (a cholinergic muscarinic receptor antagonist) impairs both the acquisition of the passive avoidance response and the retention of the learned response and that physostigmine (an acetylcholinesterase inhibitor that blocks the breakdown of the ACh released at the synaptic site) rescues the amnesic effects of scopolamine. Altogether, these findings could facilitate the use of the zebrafish as a model for the study of cholinergic mechanisms underlying learning and memory [74]. Moreover, studies related to the development of memory have been conducted in zebrafish; the data indicate that nicotine affects the memory of these organisms, similar to what has been reported in mammalian models [75]. Thus, this fish can be used to help understand the molecular mechanisms of the cholinergic system's influence on cognitive functions.

Fishes also have been used to evaluate the effects of neurotoxins, such as the case of anatoxin-a, a nicotinic agonist produced by cyanobacteria that blocks cholinergic neurotransmission to compete for the ACh receptor. This toxin can cause death in humans and other animals. Studies on rainbow trout (O. mykiss) indicate that exposure to this toxin induces increased $\mathrm{AChE}$ and lactate dehydrogenase activity, suggesting that this neurotoxin induces motor impairments and increases the metabolic demand of exposed organisms [76].

The effect of anticholinesterase pesticides has also been extensively studied in fishes. Carbofuran is a pesticide of the carbamates group that is highly toxic to mammals. In humans, this substance causes salivation, abdominal pain, chest tightness, dizziness, vomiting, and seizures. Studies on the common carp (Cyprinus carpio) indicate that this pesticide reduces the hatching rate and also induces body deformities, eye pigmentation, pericardial sac enlargement, and changes in fish behavior [77]. Also, carbofuran induces neuroendocrine dysfunctions in spotted snakehead fish (Channa punctatus) and abnormalities in the thyroid gland, possibly through an alteration of the hypothalamic-pituitary-thyroid (HPT) axis of the fish [78]. Moreover, immunotoxicity studies of this type of pesticides have shown that teleost fish comprise an excellent model for both basic research and ecotoxicology studies [79]. Studies carried out in our research group have shown that exposure to diazinon induced in Nile tilapia $(O$. niloticus) increased the RB of phagocytic cells and serum IgM concentration, but this pesticide caused a decrease in the proliferative and phagocytic capacity of leukocytes [59, 80], while chlorpyrifos, another anticholinesterase inhibitor, induced phagocytic index reduction in this fish [81]. Thus, the immunotoxic effects of anticholinesterase pesticides in vertebrates may be associated with alterations in neuronal cholinergic and extraneuronal cholinergic pathways. 


\section{Concluding Remarks}

Studies on the communication between the cholinergic and the immune systems in fish are scarce. However, this type of study could generate relevant data to contribute to the understanding of this bidirectional communication that once a full understanding of neuroendocrine control in fish has been achieved, could approach the study of bidirectional communication in evolutive terms, in addition to understanding the importance of the nonneuronal cholinergic system in nonmammalians models. These approaches certainly guarantee a better understanding of basic aspects and eventually allow the proposal of pharmacological alternatives in clinical medicine. Thus, the use of fish as a biomedical research model could contribute to a better understanding of neuroimmunomodulation mechanisms in vertebrates.

\section{Conflict of Interests}

There is no conflict of interests, and the authors declare that they have no direct relationship with the previously mentioned commercial entities or any other related.

\section{Acknowledgments}

This work was funded by a grant from the financial resources of SEP-CONACyT-México for Basic Research (Project no. 2012-179508) to M. I. Girón-Pérez and by grants from CONACyT-México 2012-180268 and PROMEP/103.5/12/8143 to A. E. Rojas-Mayorquín. The first author is student of Biologic-Agropecuary Sciences graduate program (CBAP) of Universidad Autónoma de Nayarit (México).

\section{References}

[1] I. A. Hurley, R. L. Mueller, K. A. Dunn et al., "A new time-scale for ray-finned fish evolution," Proceedings of the Royal Society B, vol. 274, no. 1609, pp. 489-498, 2007.

[2] J.-N. Volff, "Genome evolution and biodiversity in teleost fish," Heredity, vol. 94, no. 3, pp. 280-294, 2005.

[3] G. W. Litman, J. P. Cannon, and L. J. Dishaw, "Reconstructing immune phylogeny: new perspectives," Nature Reviews Immunology, vol. 5, no. 11, pp. 866-879, 2005.

[4] P. R. Rauta, B. Nayak, and S. Das, "Immune system and immune responses in fish and their role in comparative immunity study: a model for higher organisms," Immunology Letters, vol. 148, no. 1, pp. 23-33, 2012.

[5] C. M. Press and $\varnothing$. Evensen, "The morphology of the immune system in teleost fishes," Fish \& Shellfish Immunology, vol. 9, no. 4, pp. 309-318, 1999.

[6] P. Zwollo, S. Cole, E. Bromage, and S. Kaattari, "B cell heterogeneity in the teleost kidney: evidence for a maturation gradient from anterior to posterior kidney," Journal of Immunology, vol. 174, no. 11, pp. 6608-6616, 2005.

[7] A. Zapata, B. Diez, T. Cejalvo, C. Gutiérrez-De Frías, and A. Cortés, "Ontogeny of the immune system of fish," Fish \& Shellfish Immunology, vol. 20, no. 2, pp. 126-136, 2006.

[8] A. J. Davidson and L. I. Zon, "The 'definitive' (and 'primitive') guide to zebrafish hematopoiesis," Oncogene, vol. 23, no. 43, pp. 7233-7246, 2004.
[9] T. J. Bowden, P. Cook, and J. H. W. M. Rombout, "Development and function of the thymus in teleosts," Fish \& Shellfish Immunology, vol. 19, no. 5, pp. 413-427, 2005.

[10] L. Gao, C. He, X. Liu et al., "The innate immune-related genes in catfish," International Journal of Molecular Science, vol. 13, no. 11, pp. 14172-14202, 2012.

[11] T.-J. Chia, Y.-C. Wu, J.-Y. Chen, and S.-C. Chi, "Antimicrobial peptides (AMP) with antiviral activity against fish nodavirus," Fish \& Shellfish Immunology, vol. 28, no. 3, pp. 434-439, 2010.

[12] S. Saurabh and P. K. Sahoo, "Lysozyme: an important defence molecule of fish innate immune system," Aquaculture Research, vol. 39, no. 3, pp. 223-239, 2008.

[13] C. S. F. Bah, E. F. Fang, T. B. Ng, S. Mros, M. McConnell, and A. El-Din Ahmed Bekhit, "Purification and characterization of a rhamnose-binding chinook salmon roe lectin with antiproliferative activity toward tumor cells and nitric oxide-inducing activity toward murine macrophages," Journal of Agricultural and Food Chemistry, vol. 59, no. 10, pp. 5720-5728, 2011.

[14] B. Gisladottir, S. Gudmundsdottir, L. Brown, Z. O. Jonsson, and B. Magnadottir, "Isolation of two C-reactive protein homologues from cod (Gadus morhua L.) serum," Fish \& Shellfish Immunology, vol. 26, no. 2, pp. 210-219, 2009.

[15] H. Boshra, J. Li, and J. O. Sunyer, "Recent advances on the complement system of teleost fish," Fish \& Shellfish Immunology, vol. 20, no. 2, pp. 239-262, 2006.

[16] P. Alvarez-Pellitero, "Fish immunity and parasite infections: from innate immunity to immunoprophylactic prospects," Veterinary Immunology and Immunopathology, vol. 126, no. 3-4, pp. 171-198, 2008.

[17] M. A. Sylvie, P. Boudinot, and E. Bengtén, "Comprehensive survey and genomic characterization of Toll-Like Receptors (TLRs) in channel catfish, Ictalurus punctatus: identification of novel fish TLRs," Immunogenetics, vol. 65, no. 7, pp. 511-530, 2013.

[18] A. M. Rieger and D. R. Barreda, "Antimicrobial mechanisms of fish leukocytes," Developmental and Comparative Immunology, vol. 35, no. 12, pp. 1238-1245, 2011.

[19] D. Palić, J. Ostojić, C. B. Andreasen, and J. A. Roth, "Fish cast NETs: neutrophil extracellular traps are released from fish neutrophils,' Developmental and Comparative Immunology, vol. 31, no. 8, pp. 805-816, 2007.

[20] L. Pijanowski, L. Golbach, E. Kolaczkowska, M. Scheer, B. M. L. Verburg-van Kemenade, and M. Chadzinsk, "Carp neutrophilic granulocytes form extracellular traps via ROS-dependent and independent pathways," Fish \& Shellfish Immunology, vol. 34, no. 5, pp. 1244-1252, 2013.

[21] I. L. Leknes, "Eosinophilic granule cells and endocytic cells in intestinal wall of pearl gouramy (Anabantidae: Teleostei)," Fish \& Shellfish Immunology, vol. 23, no. 4, pp. 897-900, 2007.

[22] A. E. Ellis, "Innate host defense mechanisms of fish against viruses and bacteria," Developmental and Comparative Immunology, vol. 25, no. 8-9, pp. 827-839, 2001.

[23] K. J. Laing and J. D. Hansen, "Fish T cells: recent advances through genomics," Developmental and Comparative Immunology, vol. 35, no. 12, pp. 1282-1295, 2011.

[24] P. Zwollo, "Dissecting teleost B cell differentiation using transcription factors," Developmental and Comparative Immunology, vol. 35, no. 9, pp. 898-905, 2011.

[25] M. Barr, K. Mott, and P. Zwollo, "Defining terminally differentiating $\mathrm{B}$ cell populations in rainbow trout immune tissues using the transcription factor XbpI," Fish \& Shellfish Immunology, vol. 31, no. 6, pp. 727-735, 2011. 
[26] J. O. Sunyer, "Fishing for mammalian paradigms in the teleost immune system," Nature Immunology, vol. 14, pp. 320-326, 2013.

[27] H. Dooley and M. F. Flajnik, "Antibody repertoire development in cartilaginous fish," Developmental and Comparative Immunology, vol. 30, no. 1-2, pp. 43-56, 2006.

[28] L. Tort, J. C. Balasch, and S. Mackenzie, "Fish immune system. A crossroads between innate and adaptive responses," Inmunologia, vol. 22, no. 3, pp. 277-286, 2003.

[29] R. Castro, D. Bernard, M. P. Lefranc, A. Six, A. Benmansour, and P. Boudinot, "T cell diversity and TcR repertoires in teleost fish,” Fish \& Shellfish Immunology, vol. 31, no. 5, pp. 644-654, 2011.

[30] T. Wang, B. Gorgoglione, T. Maehr et al., "Fish Suppressors of Cytokine Signaling (SOCS): gene discovery, modulation of expression and function," Journal of Signal Transduction, vol. 2011, Article ID 905813, 20 pages, 2011.

[31] B. M. L. Verburg-van Kemenade, C. M. S. Ribeiro, and M. Chadzinska, "Neuroendocrine-immune interaction in fish: differential regulation of phagocyte activity by neuroendocrine factors," General and Comparative Endocrinology, vol. 172, no. 1, pp. 31-38, 2011.

[32] F. J. Arenzana, D. Clemente, R. Sánchez-González, A. Porteros, J. Aijón, and R. Arévalo, "Development of the cholinergic system in the brain and retina of the zebrafish," Brain Research Bulletin, vol. 66, no. 4-6, pp. 421-425, 2005.

[33] N. C. Bols, J. L. Brubacher, R. C. Ganassin, and L. E. J. Lee, "Ecotoxicology and innate immunity in fish," Developmental and Comparative Immunology, vol. 25, no. 8-9, pp. 853-873, 2001.

[34] T. Galloway and R. Handy, "Immunotoxicity of organophosphorous pesticides," Ecotoxicology, vol. 12, no. 1-4, pp. 345-363, 2003.

[35] Y. Abreu-Villaça, C. C. Filgueiras, and A. C. Manhães, "Developmental aspects of the cholinergic system," Behavioural Brain Research, vol. 221, no. 2, pp. 367-378, 2011.

[36] I. Wessler, C. J. Kirkpatrick, and K. Racké, "The cholinergic 'pitfall': acetylcholine, a universal cell molecule in biological systems, including humans," Clinical and Experimental Pharmacology and Physiology, vol. 26, no. 3, pp. 198-205, 1999.

[37] D. Clemente, Á. Porteros, E. Weruaga et al., "Cholinergic elements in the zebrafish central nervous system: histochemical and immunohistochemical analysis," Journal of Comparative Neurology, vol. 474, no. 1, pp. 75-107, 2004.

[38] K. Funakoshi and M. Nakano, "The sympathetic nervous system of anamniotes," Brain, Behavior and Evolution, vol. 69, no. 2, pp. 105-113, 2007.

[39] P. E. Phelps, R. P. Barber, and J. E. Vaughn, “Embryonic development of choline acetyltransferase in thoracic spinal motor neurons: somatic and autonomic neurons may be derived from a common cellular group," Journal of Comparative Neurology, vol. 307, no. 1, pp. 77-86, 1991.

[40] K. Funakoshi, Y. Atobe, T. Hisajima et al., "Choline acetyltransferase immunoreactive sympathetic ganglion cells in a teleost, Stephanolepis cirrhifer," Autonomic Neuroscience: Basic and Clinical, vol. 99, no. 1, pp. 31-39, 2002.

[41] I. Rodríguez-Moldes, P. Molist, F. Adrio et al., "Organization of cholinergic systems in the brain of different fish groups: a comparative analysis," Brain Research Bulletin, vol. 57, no. 3-4, pp. 331-334, 2002.

[42] D. Clemente, F. J. Arenzana, R. Sánchez-González, Á. Porteros, J. Aijón, and R. Arévalo, "Comparative analysis of the distribution of choline acetyltransferase in the central nervous system of cyprinids," Brain Research Bulletin, vol. 66, no. 4-6, pp. 546549, 2005.

[43] T. Mueller, P. Vernier, and M. F. Wullimann, “The adult central nervous cholinergic system of a neurogenetic model animal, the zebrafish Danio rerio," Brain Research, vol. 1011, no. 2, pp. 156169, 2004.

[44] E. P. Rico, D. B. Rosemberg, K. J. Seibt, K. M. Capiotti, R. S. Da Silva, and C. D. Bonan, "Zebrafish neurotransmitter systems as potential pharmacological and toxicological targets," Neurotoxicology and Teratology, vol. 33, no. 6, pp. 608-617, 2011.

[45] S. Steele, V. Li, A. Lo, H. Cheng, and S. Perry, "The role of the M2 muscarinic receptor in the development of hypoxic bradycardia in zebrafish (Danio rerio) larvae," Comparative Biochemistry and Physiology A, vol. 146, supplement 4, p. S182, 2007.

[46] E. D. Levin, Z. Bencan, and D. T. Cerutti, "Anxiolytic effects of nicotine in zebrafish," Physiology \& Behavior, vol. 90, no. 1, pp. 54-58, 2007.

[47] Z. Bencan and E. D. Levin, "The role of $\alpha 7$ and $\alpha 4 \beta 2$ nicotinic receptors in the nicotine-induced anxiolytic effect in zebrafish," Physiology \& Behavior, vol. 95, no. 3, pp. 408-412, 2008.

[48] C. R. Dias Assis, A. Guedes Linhares, V. M. Oliveira et al., "Comparative effect of pesticides on brain acetylcholinesterase in tropical fish," Science of the Total Environment, vol. 441, pp. 141-150, 2012.

[49] B. M. L. Verburg-Van Kemenade, E. H. Stolte, J. R. Metz, and M. Chadzinska, "Neuroendocrine-immune interactions in teleost fish," in Fish Physiology, S. D. McCormick, A. P. Farrell, and C. J. Brauner, Eds., vol. 28, chapter 7, pp. 313-364, 2009.

[50] A. S. Balasubramanian and C. D. Bhanumathy, "Noncholinergic functions of cholinesterases," FASEB Journal, vol. 7, no. 14, pp. 1354-1358, 1993.

[51] E. Weitnauer, A. Robitzki, and P. G. Layer, "Aryl acylamidase activity exhibited by butyrylcholinesterase is higher in chick than in horse, but much lower than in fetal calf serum," Neuroscience Letters, vol. 254, no. 3, pp. 153-156, 1998.

[52] L. Pezzementi and A. Chatonnet, "Evolution of cholinesterases in the animal kingdom," Chemico-Biological Interactions, vol. 187, no. 1-3, pp. 27-33, 2010.

[53] F. Ferriere, N. A. Khan, J.-P. Meyniel, and P. Deschaux, "Characterisation of serotonin transport mechanisms in rainbow trout peripheral blood lymphocytes: role in PHA-induced lymphoproliferation," Developmental and Comparative Immunology, vol. 23, no. 1, pp. 37-50, 1999.

[54] C. M. Flory, "Phylogeny of neuroimmunoregulation: effects of adrenergic and cholinergic agents on the in vitro antibody response of the rainbow trout, Onchorynchus mykiss," Developmental and Comparative Immunology, vol. 14, no. 3, pp. 283294, 1990.

[55] C. M. Flory and C. J. Bayne, "The influence of adrenergic and cholinergic agents on the chemiluminescent and mitogenic responses of leukocytes from the rainbow trout, Oncorhynchus mykiss," Developmental and Comparative Immunology, vol. 15, no. 3, pp. 135-142, 1991.

[56] S. Nilsson and D. J. Grove, "Adrenergic and cholinergic innervation of the spleen of the cod: Gadus morhua," European Journal of Pharmacology, vol. 28, no. 1, pp. 135-143, 1974.

[57] R. Fange and S. Nilsson, "The fish spleen: structure and function," Experientia, vol. 41, no. 2, pp. 152-158, 1985.

[58] M. Dunier, A. K. Siwicki, and A. Demael, "Effects of organophosphorus insecticides: effects of trichlorfon and dichlorvos on the immune response of carp (Cyprinus carpio). III. In 
vitro effects on lymphocyte proliferation and phagocytosis and in vivo effects on humoral response," Ecotoxicology and Environmental Safety, vol. 22, no. 1, pp. 79-87, 1991.

[59] M. I. Girón-Pérez, A. Santerre, F. Gonzalez-Jaime et al., "Immunotoxicity and hepatic function evaluation in Nile tilapia (Oreochromis niloticus) exposed to diazinon," Fish \& Shellfish Immunology, vol. 23, no. 4, pp. 760-769, 2007.

[60] M. I. Girón-Pérez, G. Zaitseva, J. Casas-Solis, and A. Santerre, "Effects of diazinon and diazoxon on the lymphoproliferation rate of splenocytes from Nile tilapia (Oreochromis niloticus): the immunosuppresive effect could involve an increase in acetylcholine levels," Fish \& Shellfish Immunology, vol. 25, no. 5, pp. 517-521, 2008.

[61] Y. Horiuchi, R. Kimura, N. Kato et al., "Evolutional study on acetylcholine expression," Life Sciences, vol. 72, no. 15, pp. 17451756, 2003.

[62] T. Fujii and K. Kawashima, "An independent non-neuronal cholinergic system in lymphocytes," Japanese Journal of Pharmacology, vol. 85, no. 1, pp. 11-15, 2001.

[63] K. Kawashima and T. Fujii, "The lymphocytic cholinergic system and its contribution to the regulation of immune activity," Life Sciences, vol. 74, no. 6, pp. 675-696, 2003.

[64] K. Kawashima, T. Fujii, Y. Moriwaki, H. Misawa, and K. Horiguchi, "Reconciling neuronally and nonneuronally derived acetylcholine in the regulation of immune function," Annals of the New York Aacademy of Sciences, vol. 1261, pp. 7-17, 2012.

[65] C. Reardon, G. S. Duncan, A. Brüstle et al., "Lymphocytederived ACh regulates local innate but not adaptive immunity," Annals of the New York Academy of Sciences, vol. 110, no. 4, pp. 1410-1415, 2013.

[66] S. Neumann, M. Razen, P. Habermehl et al., "The non-neuronal cholinergic system in peripheral blood cells: effects of nicotinic and muscarinic receptor antagonists on phagocytosis, respiratory burst and migration," Life Sciences, vol. 80, no. 24-25, pp. 2361-2364, 2007.

[67] Y. Okuma and Y. Nomura, "Roles of muscarinic acetylcholine receptors in interleukin-2 synthesis in lymphocytes," Japanese Journal of Pharmacology, vol. 85, no. 1, pp. 16-19, 2001.

[68] M. Behra, X. Cousin, C. Bertrand et al., "Acetylcholinesterase is required for neuronal and muscular development in the zebrafish embryo," Nature Neuroscience, vol. 5, no. 2, pp. 111-118, 2002.

[69] L. E. Hightower and J. L. Renfro, "Recent applications of fish cell culture to biomedical research," Journal of Experimental Zoology, vol. 248, no. 3, pp. 290-302, 1988.

[70] R. N. Winn, "Transgenic fish as models in environmental toxicology," ILAR Journal, vol. 42, no. 4, pp. 322-329, 2001.

[71] A. R. Cossins and D. L. Crawford, "Fish as models for environmental genomics," Nature Reviews Genetics, vol. 6, no. 4, pp. 324-333, 2005.

[72] J. Keesey, "How electric fish became sources of acetylcholine receptor," Journal of the History of the Neurosciences, vol. 14, no. 2, pp. 149-164, 2005.

[73] M. Zouridakis, P. Zisimopoulou, K. Poulas, and S. J. Tzartos, "Recent advances in understanding the structure of nicotinic acetylcholine receptors," IUBMB Life, vol. 61, no. 4, pp. 407-423, 2009.

[74] S. Santana, E. P. Rico, and J. S. Burgos, "Can zebrafish be used as animal model to study Alzheimer's disease?" American Journal of Neurodegenerative Diseases, vol. 1, no. 1, pp. 32-48, 2012.
[75] E. D. Levin and E. Chen, "Nicotinic involvement in memory function in zebrafish," Neurotoxicology and Teratology, vol. 26, no. 6, pp. 731-735, 2004.

[76] J. Osswald, A. P. Carvalho, L. Guimarães, and L. Guilhermino, "Toxic effects of pure anatoxin-a on biomarkers of rainbow trout, Oncorhynchus mykiss," Toxicon, vol. 70, pp. 162-169, 2013.

[77] K. R. Pawar, "Toxic of teratogenic effect of fenitrothion, BHC and carbofuran on the embryonic development of Cyprinus carpio communis," Environment and Ecology, vol. 12, no. 2, pp. 284-287, 1994.

[78] R. N. Ram and S. K. Singh, "Carbofuran-induced histopathological and biochemical changes in liver of the teleost fish, Channa punctatus (Bloch)," Ecotoxicology and Environmental Safety, vol. 16, no. 3, pp. 194-201, 1988.

[79] J. T. Zelikoff, "Biomarkers of immunotoxicity in fish and other non-mammalian sentinel species: predictive value for mammals?" Toxicology, vol. 129, no. 1, pp. 63-71, 1998.

[80] M. I. Girón-Pérez, J. Velázquez-Fernández, K. Díaz-Resendiz et al., "Immunologic parameters evaluations in Nile tilapia (Oreochromis niloticus) exposed to sublethal concentrations of diazinon," Fish \& Shellfish Immunology, vol. 27, no. 2, pp. 383385, 2009.

[81] M. I. Girón-Pérez, R. Barcelós-García, Z. G. Vidal-Chavez, C. A. Romero-Bañuelos, and M. L. Robledo-Marenco, "Effect of chlorpyrifos on the hematology and phagocytic activity of Nile tilapia cells (Oreochromis niloticus)," Toxicology Mechanisms and Methods, vol. 16, no. 9, pp. 495-499, 2006. 


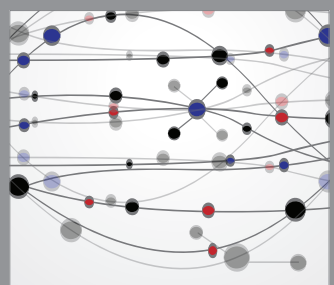

The Scientific World Journal
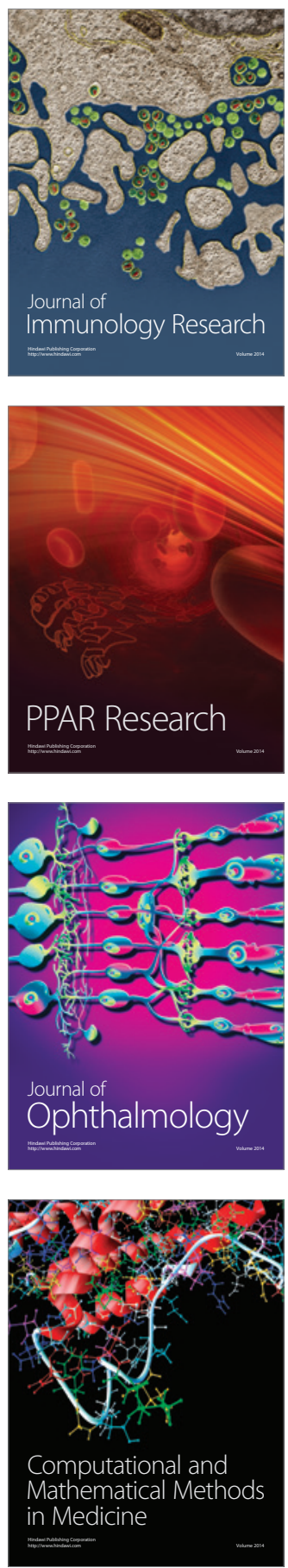

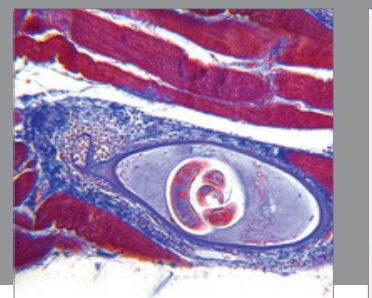

Gastroenterology

Research and Practice
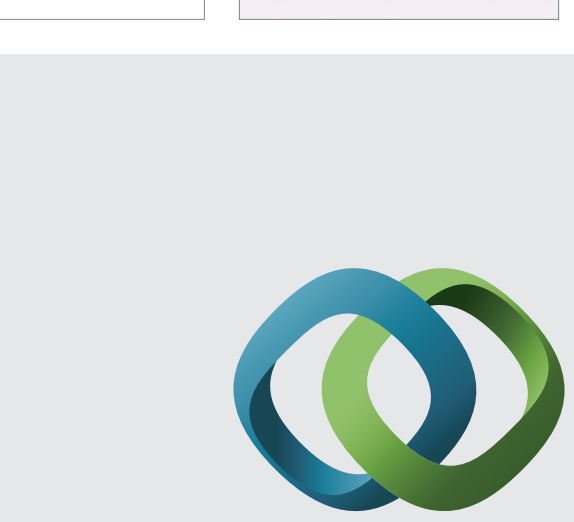

\section{Hindawi}

Submit your manuscripts at

http://www.hindawi.com
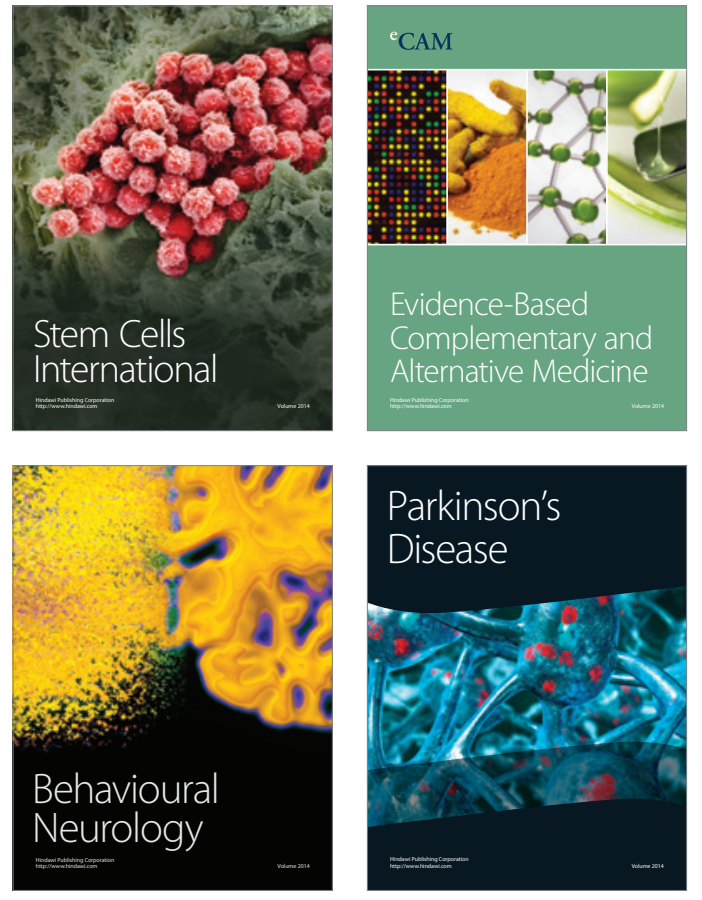
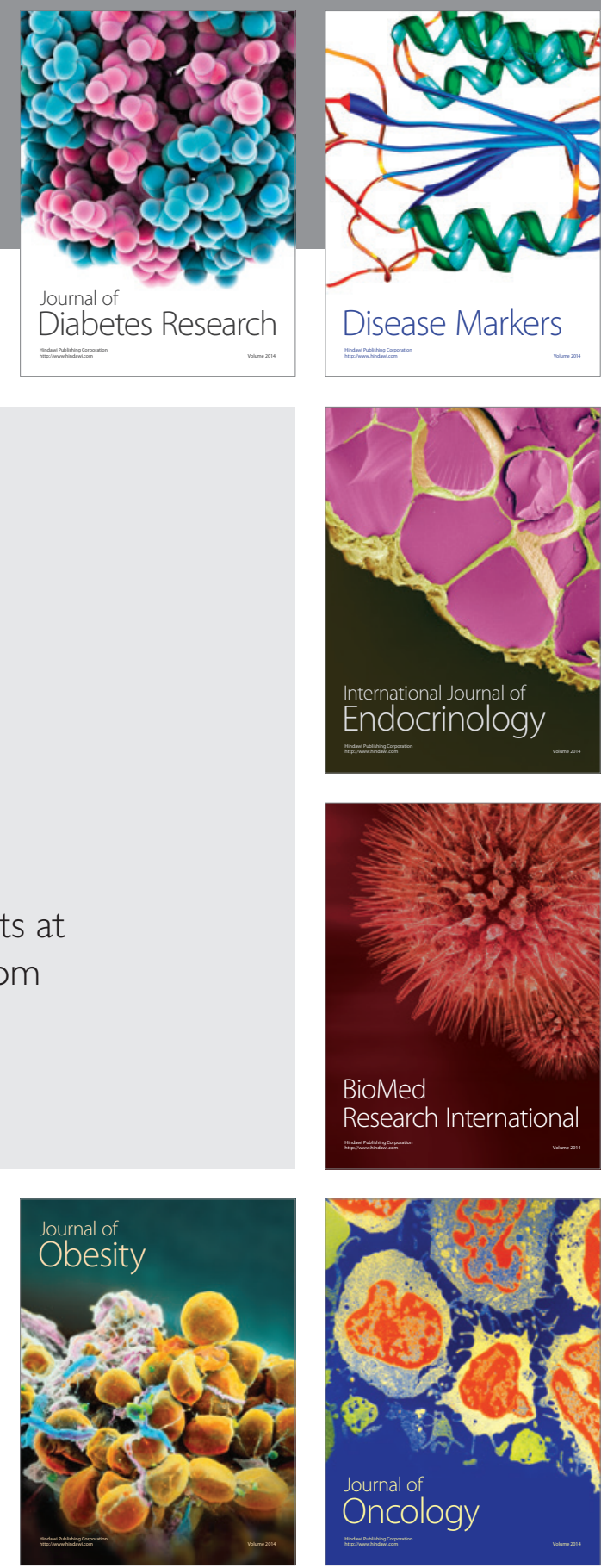

Disease Markers
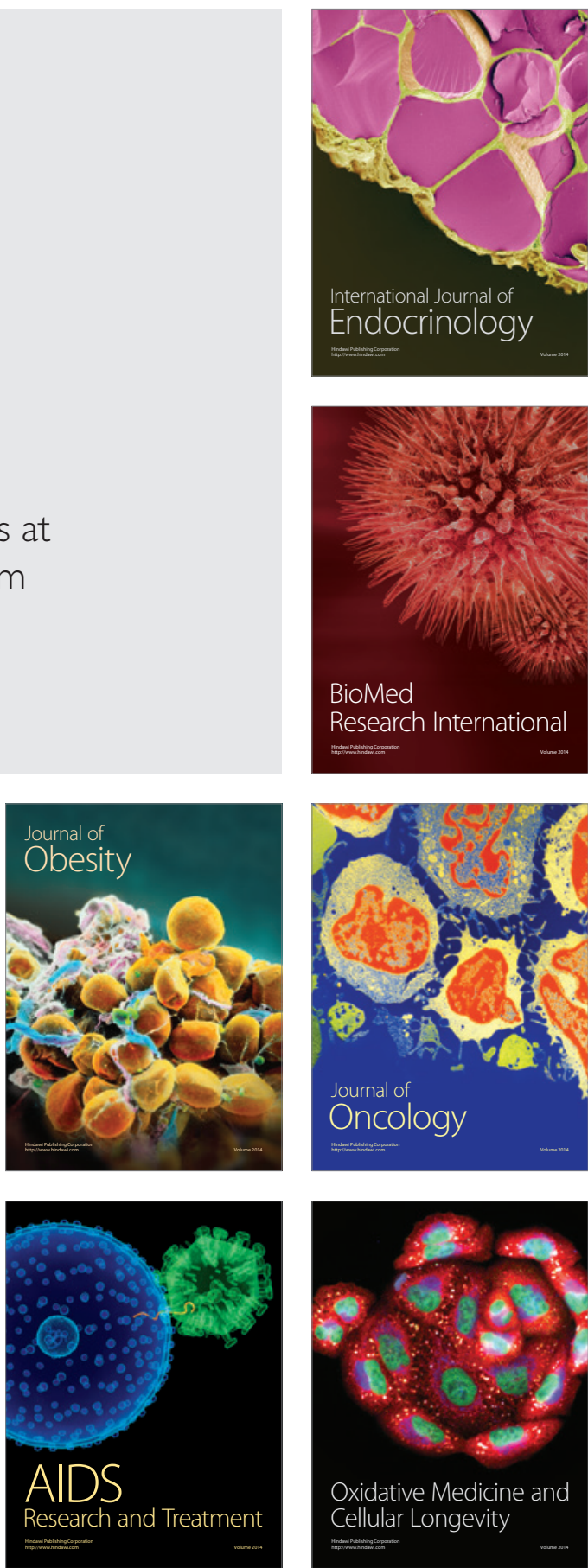